\title{
Rats' performance on an interval time-place task: Increasing sequence complexity
}

\author{
CHRISTINA M. THORPE and DONALD M. WILKIE \\ University of British Columbia, Vancouver, British Columbia, Canada
}

\begin{abstract}
Rats were trained on an interval time-place learning (TPL) task in which the location of food availability depended on the time since the start of the session. Each of four levers (numbered 1, 2, 3, 4) provided food on an intermittent schedule for two nonconsecutive 3-min periods. The order in which the levers provided food was 1, 2, 4, 3, 2, 3, 1, 4. This order was consistent across sessions. Previous research conducted in our lab has shown that when only four "places" are used, rather than the eight in the present study, rats use a timing strategy to track the location of food. Pizzo and Crystal (2004) recently trained rats on an interval TPL in which each of eight arms of a radial arm maze provided food. They found evidence suggesting that rats used both spatial and temporal information. In the present study, in which a revisiting strategy was used (i.e., each lever provided food on more than one occasion), the rats tracked both the spatial and the temporal availability of food for the first half of the session. Interestingly, in the second half of the sessions, the rats appeared to be timing the availability of food even though they did not know where it would occur. That is, the rats knew the temporal, but not the spatial, contingencies for the second half of the session. It appears that the requirement of revisiting a previously reinforced lever resulted in rats' no longer being able to solve the spatial aspect of the task.
\end{abstract}

The ability to learn the spatiotemporal variability of biologically significant stimuli is known as time-place learning (TPL). This ability presumably confers an advantage to an animal by allowing it to plan the most efficient way to obtain access to stimuli such as food and mates, and to avoid predators. Some researchers have suggested that time-place information is so important to an animal that it likely forms the basis of how memories are encoded. For example, Gallistel (1990) posits that whenever a biologically significant event occurs, a tripartite memory code is made that includes the time, place, and nature of the event. When the animal is later faced with a similar biological need, it can consult these memory codes and determine when and where that need has been met in the past.

There are at least two types of TPL: daily and interval. In daily TPL, the location of some stimuli (usually food) is dependent on the time of day. For example, Biebach, Gordijn, and Krebs (1989) successfully trained garden warblers to go to one of four rooms in a testing apparatus, depending on the time of day for access to a food reward. This ability has also been demonstrated in honeybees (Wahl, 1932, as cited in Reebs, 1993), ants (Schatz, Beugnon, \& Lachaud, 1994; Schatz, Lachaud, \& Beugnon, 1999), pigeons (Saksida

This research was supported by the Natural Sciences and Engineering Research Council of Canada. Daniela Meier, Jane Sun, and Jessica Otte helped with data collection. These data constituted part of C.M.T.'s $\mathrm{PhD}$ dissertation. Correspondence should be addressed to $\mathrm{C}$. M. Thorpe, Department of Psychology, University College London, Gower Street, London WC1E 6BT, England (e-mail: christina.thorpe@ucl.ac.uk).
\& Wilkie, 1994), fish (Reebs, 1999), and rats (Widman, Gordon, \& Timberlake, 2000; but see Thorpe, Bates, \& Wilkie, 2003).

The second type of TPL, interval TPL, forms the basis of the present study. In interval TPL, the location of a stimulus depends on the amount of time that has passed since the onset of some other stimulus. In the first published paper on interval TPL, Wilkie and Willson (1992) trained pigeons in a transparent operant chamber that contained a key and a food hopper on each of its four walls. Responses on the keys were reinforced according to a time-place contingency. The first keylight provided food on an intermittent schedule for $15 \mathrm{~min}$, followed by the second keylight for the next $15 \mathrm{~min}$, and then by the third and fourth keylights also for $15 \mathrm{~min}$ each. Only one key provided reinforcement at any given time, and the order in which the keys provided reinforcement remained constant from session to session. The pigeons were able to successfully learn this time-place contingency. Similar studies have also been conducted with rats (e.g., Carr \& Wilkie, 1998; Thorpe, Floresco, Carr, \& Wilkie, 2002).

A number of similarities exist between rats and pigeons trained on these tasks. First, both restrict the majority of their responding to the key or lever that currently provides food. Second, both have a tendency to anticipate the occurrence of food, shown in their tendency to start responding on the key or lever before it actually starts providing food. Third, both have a tendency to anticipate depletion of reinforcement, shown in their tendency to decrease responding on the key or lever just prior to when it actually stops providing food. These three findings strongly 
suggest that the animals are timing the spatiotemporal movement of the reinforced key or lever. More concrete evidence for this claim comes from probe trials (known as open hopper trials, OHTs). In these probe trials, no time-place contingencies are in effect. That is, all levers provide reinforcement for the entire session. OHTs are administered once the animal has mastered the TPL task, to provide convincing evidence that the rats are timing rather than relying solely on some other strategy such as discrimination of when a lever is, and is not, providing food. It would be somewhat difficult (but not impossible) to detect changes in rate of reinforcement on a lever, because the levers provide food on a variable ratio schedule. According to this win-stay lose-shift strategy, the rats could simply press one lever until it failed to give them food and then search for the next lever providing food. Numerous studies from our laboratory have shown that during OHTs, animals continue to switch to the next lever at approximately the correct time, despite there being no contingencies in effect to necessitate their doing so (e.g., Carr, Tan, Thorpe, \& Wilkie, 2001; Thorpe, Floresco, et al., 2002; Thorpe, Petrovic, \& Wilkie, 2002). The error is slightly greater on OHTs than on baseline sessions. This is probably due to the fact that in addition to timing, the rats also use the presence or absence of food as a cue that their timing is accurate. If on OHTs the rats do not move from lever to lever at approximately the correct time, this is an indication that the rats are not using timing as their primary means of solving the TPL task.

In previous interval TPL tasks, two characteristics have always been consistent. First, the duration for which the levers provided food was constant throughout the entire session. For example, all levers provided food for $4 \mathrm{~min}$. Second, there was a maximum of four places. Recently, Crystal and Miller (2002) and Thorpe and Wilkie (2002) have manipulated these two factors. Thorpe and Wilkie manipulated the first of these variables by training rats on an interval TPL task in which each period was of differing duration. More specifically, the first lever provided food for $6 \mathrm{~min}$, the second for $4 \mathrm{~min}$, the third for $2 \mathrm{~min}$, and the fourth for $8 \mathrm{~min}$. Two interesting findings emerged from this study. First, the rats were able to learn this task, as was shown by the presence of anticipation on baseline sessions, as well as by their tendency to move from lever to lever on OHTs. Second, the rats' behavior on this task was not in agreement with scalar timing/Weber's law, which, as applied to timing, predicts that as the duration being timed increases, the error in timing increases proportionally. According to Gibbon (1991), there are two hallmark features of scalar timing: superposition and a constant coefficient of variation. According to the rule of superposition, the response-rate curves over time should be superimposed when both axes are normalized. The coefficient of variation is defined as the ratio of the standard deviation to the mean of the response distribution, and this ratio needs to be constant according to scalar timing. Neither of these conditions was met in Thorpe and Wilkie's (2002) study. That is, the response-rate curves were not superimposed, and the coefficient of variation was not constant.
The purpose of the present study was to determine how rats would respond to an interval TPL task in which there was an increase in the number of places. There are likely to be differences among species in their ability to time the availability of food in multiple locations. It is therefore difficult to predict how rats will respond to an interval TPL in which there is an increase in the number of locations. In nature, some animals seem to learn about only a small number of places. For example, Sibly and McCleery (1983) observed that herring gulls typically visited a grassy field in the morning and a garbage dump in the afternoon. But, on the other hand, animals such as hummingbirds visit a large number of places, staying as long as it takes to deplete the nectar in the flowers.

There are two possible ways to increase the number of the places in a TPL paradigm: increase the physical number of locations (e.g., have eight levers) or require the rats to visit the same place more than once (i.e., use a revisiting strategy). Pizzo and Crystal (2004) recently conducted an experiment in which rats were trained on an interval TPL task in which each of eight arms of a radial maze provided food on an intermittent schedule for $7 \mathrm{~min}$. The rats successfully learned to go to the correct arm at the correct time. Furthermore, evidence of anticipation suggests that the rats were using a timing strategy to solve the task (although no OHTs were conducted). In the present study, we increased the number of places by using the second strategy - that is, by requiring rats to revisit locations. The rats were trained on an interval TPL task in which each lever provided food for $3 \mathrm{~min}$ on a variable ratio 15 (VR15) schedule at two different times. The order in which the levers provided food was chosen in a semirandom manner such that one lever did not follow the same lever on more than one occasion and such that the same lever was not active for two consecutive periods. The order was 1, 2, 4, 3, 2, 3, 1, 4 and was consistent across rats and sessions.

\section{METHOD}

\section{Subjects and Apparatus}

The subjects were 4 experimentally naive male Long Evans rats acquired from Charles River (St. Constant, QC). At the beginning of the experiment, they were approximately 100 days old. The rats were maintained at approximately $90 \%$ of their free-feeding weights, adjusted for age. To maintain their weights, the rats received standard rat diet (PMI Feeds, Richmond, IN) at the end of test days and on nontest days. During test sessions, the rats received 45-mg Noyes A/I Pellets (Research Diets, New Brunswick, NJ). The rats had free access to water except during test sessions.

The rats were housed individually in large transparent, plastic cages lined with Bed o' Cobs (Andersons, Maumee, $\mathrm{OH}$ ) bedding. Each week, the rats were given paper products to build nests. The rats received four to six sessions per week of group behavioral enrichment, which consisted of being placed in a chamber containing various tubes, ladders, toys, and other rats for $20 \mathrm{~min}$ per session. The colony room was maintained on a 12:12-h light:dark cycle, with light onset at $0730 \mathrm{~h}$ and offset at $1930 \mathrm{~h}$.

The rats were tested in a Plexiglas chamber $(40 \times 40 \times 40 \mathrm{~cm})$. The chamber was located on a tabletop in a small, well-lit room. The chamber was transparent, permitting the rat to view various distal room cues. The floor of the chamber was covered with $2 \mathrm{~cm}$ of Bed o' Cobs bedding. Centered on each of the four walls, $4 \mathrm{~cm}$ 
from the floor, was a lever. A brass food cup was located next to the lever. Leverpresses were recorded by the closure of a microswitch mounted on each lever. Four pellet hoppers (Scientific Prototype Mfg. Corp., New York, NY, Model No. D700) were mounted on top of the chamber. When operated, the hoppers dispensed 45-mg Noyes $\mathrm{A} / \mathrm{I}$ reward pellets into the food cups next to the levers. A small cue light (28 V DC) was mounted above each lever.

\section{Procedure}

The rats were initially placed in groups of 3 or 4 in the testing chamber. During this phase, all four of the levers provided food reinforcement on a continuous reinforcement (CRF) schedule. The levers were coated with a food mash mixture, to encourage the rats to approach the levers. Once all rats were consistently lever pressing, they began individual training. During individual training, the rats were gradually shifted from the CRF schedule to a variable ratio VR15 schedule (i.e., on average, every 15 th response is reinforced).

When the rats were consistently pressing on the VR 15 schedule, they began training on the TPL task. The rats received two sessions per day. The first session was conducted from 1 to $3 \mathrm{~h}$ after colony light onset. The second session was conducted from 2 to $3 \mathrm{~h}$ after the completion of the first session. The rats were tested 5 to 6 days per week. By the completion of the experiment, the rats had received approximately 350 sessions. The order in which the rats were tested varied randomly across days. The rats were placed into the testing chamber and a 1-min nonreinforcement period began. Responses had no consequences during this time. The purpose of this nonreinforcement period was to allow the rats sufficient time to patrol and inspect the chamber and food sites before testing began. After this 1-min nonreinforced period, the cue lights came on, and the session proper began. (For a more detailed discussion of this nonreinforced period, see Wilkie, Willson, \& Carr, 1999.)

Test sessions were $24 \mathrm{~min}$ long and were divided into eight periods of equal length. During each period, only one lever provided reward pellets according to a VR15 schedule. The levers provided food in the same order for all rats in all sessions. The levers were successively numbered $1-4$, and the order in which they provided reinforcement was 1, 2, 4, 3, 2, 3, 1, 4. The timing of each leverpress and reward delivery was recorded to 1 -sec accuracy on a remote server computer. A data file was generated in which the time at which each response occurred was recorded, in addition to which lever was pressed, and whether the response resulted in the delivery of a food pellet.

In addition to baseline sessions, the rats also received three OHT sessions interspersed between baseline sessions. The OHT sessions were conducted on Sessions 316, 326, and 342 for 2 rats; on Sessions 329, 339, and 354 for the 3rd rat; and on Sessions 325, 335, and 351 for the 4th rat. During the OHT sessions, all levers provided food at all times according to a VR15 schedule. As has been discussed in the introduction, the purpose of the OHTs was to provide further evidence that the rats were timing food availability.

\section{RESULTS}

Results are reported for the last 40 baseline sessions. The 24-min sessions were first broken down into fortyeight 30 -sec recording bins. The total number of responses

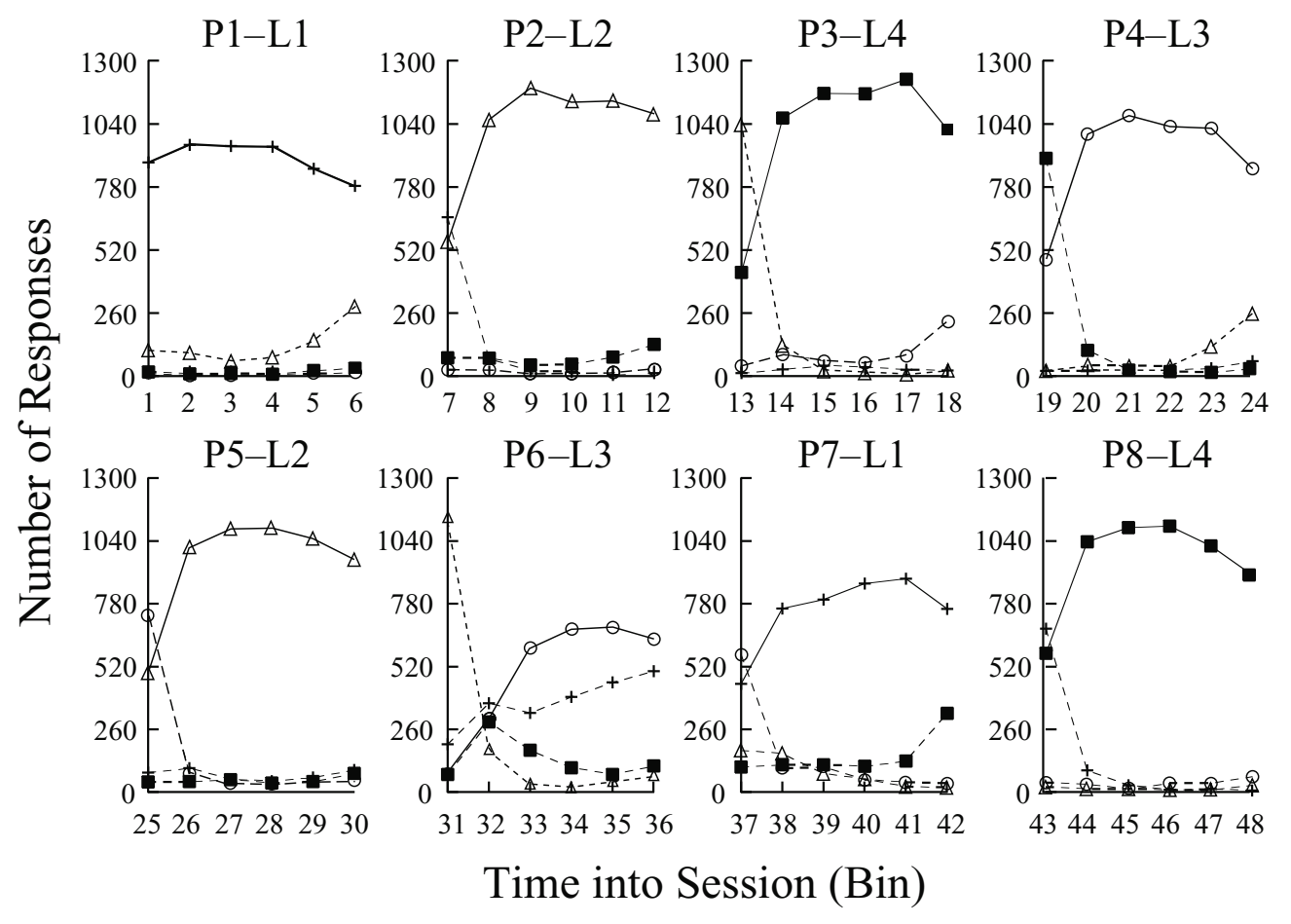

+ Lever $1 \Delta$ Lever $2 \quad$ O Lever $3 \quad$ Lever 4

Figure 1. The average response-rate distributions on Levers 1, 2,3, and 4 during the 40 baseline sessions. Each period is represented in a separate panel. The labels along the top of the graph denote the periods and levers that provided food (e.g., P3-L4 means that Lever 4 provided food during Period 3). The lever that currently provides food is represented by solid lines, whereas the levers that do not currently provide food are represented by dashed lines. 
that each rat made during the 40 sessions in each of these bins was then calculated for each of the four levers. These individual response distributions were then averaged across all 4 rats. The average response distributions are plotted in Figure 1, with each period graphed separately; it is apparent that the rats restricted the majority of responding on a lever to the periods in which it provided food (with the possible exception of Period 6; P6-L3). To confirm this impression, the percent correct responses for each period was calculated by dividing the number of responses to the lever that currently provided reinforcement by the total number of responses on all four levers during the period. The percent correct scores for each period are shown in Figure 2. One-sample $t$ tests were conducted to compare the percent correct during each period with chance levels of $25 \%$. The results are shown in Table 1 . Because multiple $t$ tests were conducted, an $\alpha$ of .01 was used. Although percent correct scores did not differ significantly from chance in either Period $6[t(3)=1.233$, $p=.306]$ or Period $7[t(3)=4.041, p=.027]$, the percent correct scores during the other periods appeared to be significantly different from chance.

To determine whether the rats were displaying anticipation (i.e., pressing on a lever just prior to when the lever started providing food) and anticipation of depletion (i.e., decreasing the amount of responding on a lever just prior to when the lever stopped providing food), response rates just before the start and end of rewarded periods were examined. Anticipation could clearly be seen in the first half of the session (i.e., Periods 1-4). For the second half of the session, however, the results were not so clear. During Periods 5 and 6, the rats were not anticipating the next correct lever. (Anticipation is the gradual increase in rate of responding on the next reinforced lever. During Period 6, the average rate of responding on the next correct lever, Lever 1, was high during the entire period. For this reason, we do not consider the rats to have been anticipating during Period 6.) They did appear to have anticipated the next lever during Period 7, and because Period 8 was the last lever in the session there was no next lever to anticipate. There was evidence of anticipation of depletion for all the periods.

Because the anticipation and anticipation of depletion data did not permit conclusive determination of whether or not the rats were timing food availability, the results of the three OHT sessions were analyzed. These data were analyzed in the same manner as were the baseline data. The average response distributions for the OHT sessions are shown in Figure 3: During the first half of the session (i.e., Periods 1-4), the rats appear to have moved from lever to lever at approximately the correct time. During the second half of the session (i.e., Periods 5-8), however, the "errors" substantially increased. To quantify this trend, the percentage of "correct" choices during each period was computed. On OHTs, all levers provide reinforcement during all periods, so technically all responses are correct. However, when we say "correct responses in the context of OHTs," we mean responding on the lever that would have been correct on baseline sessions when the contingencies were in place. The percent correct scores for each period during OHTs are shown in Figure 2. Again the percent correct scores were compared with chance in one-sample $t$ tests, and the results are presented in Table 1. Percent correct was significantly different from chance for Periods 1 and 2 . For subsequent periods, the results were only marginally significant or nonsignificant.

The pattern of results in Figure 2 is worth commenting on briefly. First, we can see that the percent correct scores are lower for the OHT than for the baseline sessions. To confirm this statistically, a 2 (session type: baseline vs. OHT) $\times 8$ (period) repeated measures ANOVA was conducted. There were significant main effects of period $[F(7,21)=22.180, p<.001]$ and session type $[F(1,3)=$ $24.192, p=.016]$, as well as a significant session type $\times$ period interaction $[F(7,21)=3.947, p=.007]$. Pairedsample $t$ tests were then conducted as a follow-up. A comparison of percent correct scores on baseline and OHT sessions showed significant differences between the two

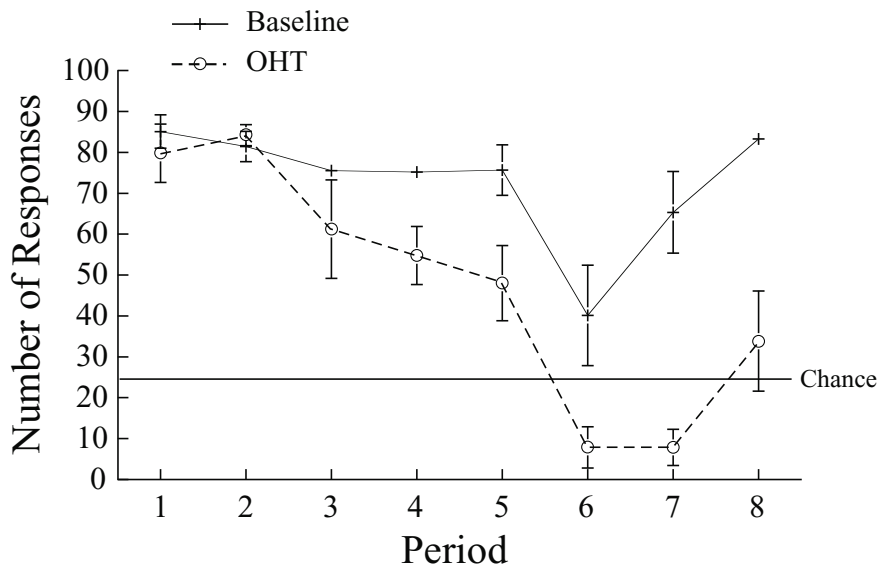

Figure 2. The percent correct responses during each period on both baseline and open hopper trial (OHT) probe tests. The horizontal line represents chance level. Error bars represent standard errors of the means. 


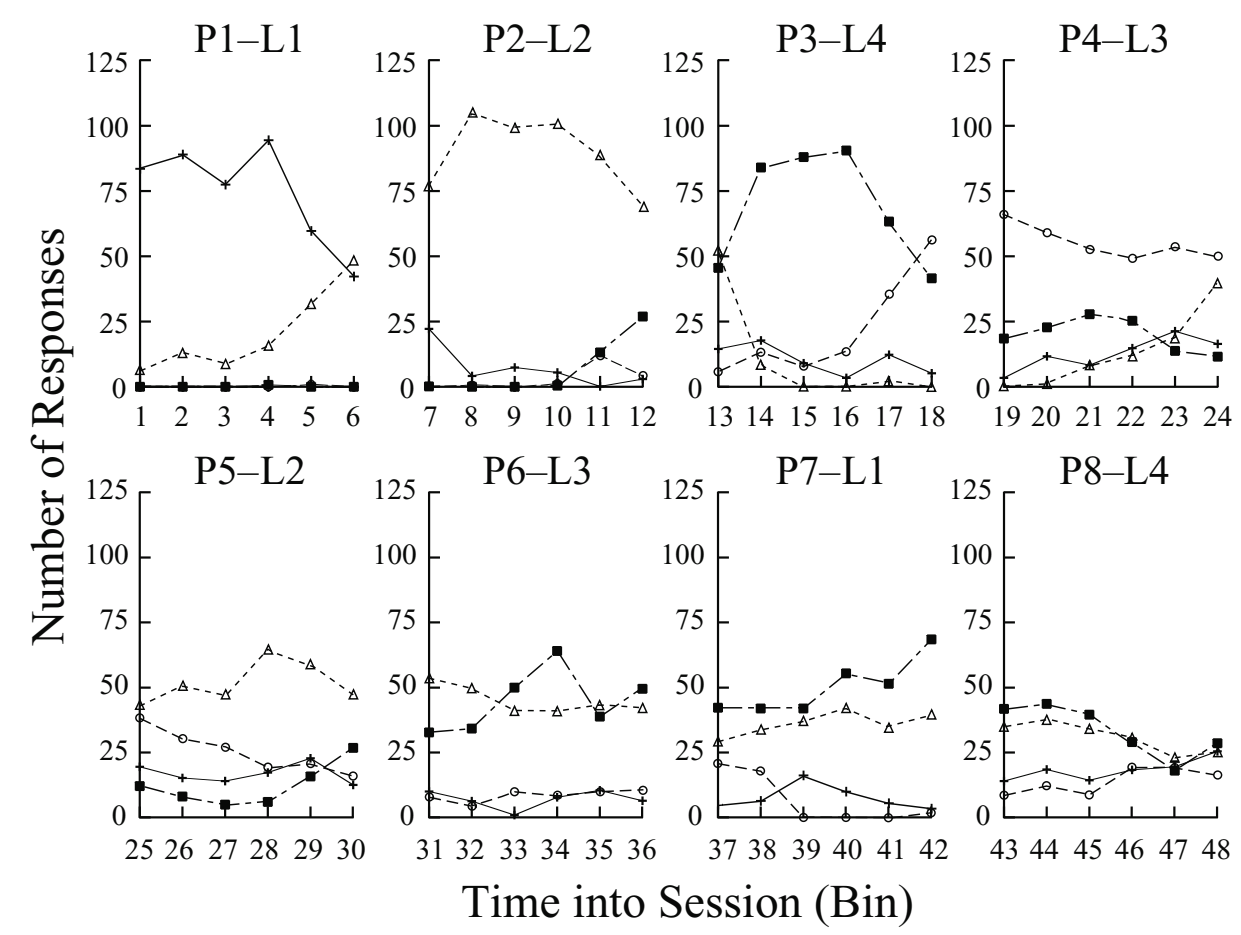

+ Lever $1 \Delta$ Lever $2 \quad$ O Lever $3 \quad$ Lever 4

Figure 3. The average response-rate distributions on Levers 1,2,3, and 4 during the three open hopper trial sessions. Each period is represented in a separate panel. The labels along the top of the graph denote the periods and levers that provided food (e.g., P3-L4 means that Lever 4 provided food during Period 3). The levers that provided food during baseline sessions are represented by solid lines, whereas the levers that did not provide food during baseline sessions are represented by dashed lines.

on Periods 7 and $8[t(3)=4.805, p=.017$, and $t(3)=$ $4.587, p=.019$, respectively] and a trend toward significance for Periods 4 and $5[t(3)=2.946, p=.060$, and $t(3)=2.882, p=.063$, respectively]. It thus appears that there were no significant differences between the baseline and OHT percent correct scores during the first few periods and that the percent correct scores tended to diverge toward the end of the session.

Second, if we had not imposed a more stringent $\alpha$ of .01 to accommodate the number of $t$ tests conducted, but

Table 1

Results of One-Sample $t$ Tests Comparing Percent Correct Score on Each Period to Chance Levels of $25 \%$

\begin{tabular}{crrrrr}
\hline & \multicolumn{3}{c}{ Score on Each Period to Chance Levels of 25\% } \\
\cline { 2 - 3 } \cline { 5 - 6 } Period & \multicolumn{2}{c}{ Baseline } & & \multicolumn{2}{c}{ OHT } \\
\cline { 2 - 3 } \cline { 5 - 6 } & 14.623 & .001 & & \multicolumn{1}{c}{$t .663$} & .005 \\
2 & 15.348 & .001 & & 22.923 & $<.001$ \\
3 & 37.045 & $<.001$ & & 3.005 & .057 \\
4 & 37.463 & $<.001$ & & 4.193 & .025 \\
5 & 8.218 & .004 & & 2.509 & .087 \\
6 & 1.233 & .306 & & -3.401 & .042 \\
7 & 4.041 & .027 & & -3.847 & .031 \\
8 & 38.519 & $<.001$ & & 0.721 & .523 \\
\hline
\end{tabular}

Note-OHT, open hopper trial(s). In all cases, $d f=3$. instead had used the standard $\alpha$ level of .05 , the percent correct scores during Periods 6 and 7 would have been statistically below chance levels. Given that we have such a small sample size, continuing to use $\alpha$ of .05 may not have been an unreasonable option. Given for a moment that Periods 6 and 7 were statistically below chance levels, why might this have been the case? During Periods 6-8, the rats restricted the majority of their responding to Levers 2 and 4. During Periods 5 and 8, these were the "correct" levers, and therefore responding on them did not adversely affect the percent correct scores as it did during Periods 6 and 7.

\section{DISCUSSION}

Rats learned to respond more on the correct levers in an interval TPL task that required revisiting of rewarded levers. Rats could solve this task simply by responding on a lever until it stopped providing food and then moving to another lever that provided food. This is a winstay/lose-shift strategy. Alternatively, the rats could use knowledge of the temporal and spatial regularities in the task to determine when and to where it should move. To determine which of these two methods was used, both the baseline and OHT data were examined. In the present 
study, examining only the baseline data lead to conflicting conclusions. During all eight periods, the rats anticipated depletion of food availability on a lever before that lever actually stopped providing food, suggesting that the rats had knowledge of the duration during which a given lever provided food. However, the anticipation of food availability data were mixed. During the first four periods, the rats appear to have anticipated the arrival of food on a lever before it actually started to provide food, suggesting that for the first half of the session the rats knew both the duration during which the food was available and the location at which it would be available. During the second half of the session, the rats did not anticipate the next lever to provide food.

Although it seems clear that the rats knew both the spatial and the temporal regularities of the food during the first half of the session (i.e., Periods 1-4), the data are not so clear for the second half of the session (i.e., Periods 5-8).To get a better sense of what the rats were doing during the second half of the session, the results of the OHTs were analyzed. The pattern of results suggests that the percent correct responses on OHTs (i.e., the percent responses on the lever that would have been correct if the baseline contingencies were in effect) were significantly above chance for the first two periods, and then decreased as the session proceeded. There was a trend toward significance for above chance responding in Periods 3-7, whereas responding during the last period was clearly nonsignificant. As well, there were significant differences between the percent correct scores on baseline trials and OHTs for Periods 7 and 8 , and a trend toward significance for Periods 4 and 5 . Note that if a more lenient $\alpha$ of .05 had been used to accommodate the small sample size, it would have been concluded that the rats responded significantly below chance levels during Periods 6 and 7 of the OHTs. As has been noted in the Results section, this probably reflected the fact that during Periods 5-8 the rats responded mostly on Levers 2 and 4, which were incorrect during Periods 6 and 7. Taken together, these results confirm our hypothesis that the rats knew the spatial and temporal contingencies in effect for the first part of the session, but that as the session proceeded, the rats no longer used spatial and temporal knowledge to solve the task.

Although the rats were not using both spatial and temporal information in the second half of the session, is it possible that they were using just one of either spatial or temporal information? It appears that the rats did not use spatial information in the second half of the session. Evidence for the claim that the rats did not know which lever would next provide reinforcement comes from the apparent increase in variability of responding during Period 6 as well as the tendency of the rats to respond mostly on Levers 2 and 4 during Periods 5-8. One could speculate that in the present study, the rats correctly learned the order in which the levers provided food for the first half of the session (i.e., Periods 1-4), but that when they were required to visit a place for the second time in the same session, there was interference from the first round. It is not surprising that there might be some degree of interference or confusion due to the fact that each lever (except Lever 1) was succeeded by two different levers.

Although the rats did not seem to know the spatial contingencies in the second half of the present study, it is possible that they had learned the temporal contingencies in effect. The fact that anticipation of depletion was seen during all periods suggests that the rats knew when a lever would stop providing food. Given that the rats did not know to which lever they should move, it is not surprising that they did not start responding on the lever prior to its giving reinforcement. This can also explain why the rats did not move to the next correct lever at the correct time on the OHTs.

The findings of the present study are in opposition to those of Pizzo and Crystal (2004). Those researchers trained rats on an interval TPL task in which each of eight arms of a radial arm maze provided food for a 7-min period. Rats trained using this design appeared to learn both the spatial and the temporal contingencies for the entire session. Unfortunately, Pizzo and Crystal did not conduct OHT probe sessions to rule out the use of a nontemporal win-stay/lose-shift strategy, although they have provided rather compelling evidence for a temporal strategy by presenting anticipation data. Why were the rats in Pizzo and Crystal's study able to learn both the spatial and the temporal contingencies? The major difference between their study and ours is that in the present study, we required the rats to use a revisiting strategy, whereas in Pizzo and Crystal's study, the rats visited each location only once during a session. It may be the case that the requirement to learn that any given lever may be followed by either of two levers, as was the case in the present study, causes interference in learning and memory of the sequence.

To determine whether or not this is the case, it would be worthwhile to replicate the present study using a repeating pattern (e.g., 1, 2, 3, 4, 1, 2, 3, 4) to verify that it is the interference of the order in which the levers provided food in the first half of the session with the order in which the levers provided food in the second half of the study that resulted in the rats' not knowing the sequence during the second half of the session. As well, the present study should be replicated using a revisiting paradigm on a radial arm maze, to ensure that the discrepancy in results was due to the requirement that rats revisit places rather than to the spatial nature of the task.

\section{REFERENCES}

Biebach, H., Gordijn, M., \& Krebs, J. (1989). Time-place learning by garden warblers, Sylvia borin. Animal Behaviour, 37, 353-360.

Carr, J. A. R., Tan, A. O., Thorpe, C. M., \& Wilkie, D. M. (2001). Further evidence of joint time-place control of rats' behaviour: Results from an "open hopper" test. Behavioural Processes, 53, 147-153.

CARR, J. A. R., \& WILKIE, D. M. (1998). Characterization of the strategy used by rats in an interval time-place learning task. Journal of Experimental Psychology: Animal Behavior Processes, 24, 151-162. 
Crystal, J. D., \& Miller, B. J. (2002). Simultaneous temporal and spatial processing. Animal Learning \& Behavior, 30, 53-65.

Gallistel, C. R. (1990). The organization of learning. Cambridge, MA: MIT Press.

GibBon, J. (1991). Origins of scalar timing. Learning \& Motivation, 22, 3-38

Pizzo, M. J., \& Crystal, J. D. (2004). Time-place learning in the eightarm radial maze. Learning \& Behavior, 23, 240-255.

ReEBs, S. G. (1993). A test of time-place learning in a cichlid fish. Behavioural Processes, 30, 273-282.

ReEbs, S. G. (1999). Time-place learning based on food but not on predation risk in a fish, the inanga (Galaxias maculates). Ethology, 105, 361-371.

SAKsIDA, L. M., \& WILKIE, D. M. (1994). Time-of-day discrimination by pigeons, Columba livia. Animal Learning \& Behavior, 22, 143-154.

Schatz, B., Beugnon, G., \& Lachaud, J. P. (1994). Time-place learning by an invertebrate, the ant Ectatomma ruidum Roger. Animal Behaviour, 48, 236-238.

Schatz, B., Lachaud, J. P., \& Beugnon, G. (1999). Spatio-temporal learning by the ant Ectatomma ruidum. Journal of Experimental Biology, 202, 1897-1907.

Sibly, R. M., \& McCleery, R. H. (1983). The distribution between feeding sites of herring gulls breeding at Walney Island, U.K. Journal of Animal Ecology, 52, 51-68.

Thorpe, C. M., BAtes, M. E., \& Wilkie, D. M. (2003). Rats have trou- ble associating all three parts of the time-place-event memory code. Behavioural Processes, 63, 95-110.

Thorpe, C. M., Floresco, S. B., Carr, J. A. R., \& Wilkie, D. M. (2002). Alterations in time-place learning induced by lesions to the rat medial prefrontal cortex. Behavioural Processes, 59, 87-100.

Thorpe, C. M., Petrovic, V., \& Wilkie, D. M. (2002). How rats process spatiotemporal information in the face of distraction. Behavioural Processes, 58, 79-90.

Thorpe, C. M., \& Wilkie, D. M. (2002). Unequal interval time-place learning. Behavioural Processes, 58, 157-166.

WAHL, O. (1932). Neue Untersuchungen über das Zeitgedächtnis der Bienen. Zeitschrift für Vergleichende Physiologie, 9, 259-338.

Widman, D. R., Gordon, D., \& Timberlake, W. (2000). Response cost and time-place discrimination by rats in maze tasks. Animal Learning \& Behavior, 28, 298-309.

Wilkie, D. M., \& Willson, R. J. (1992). Time-place learning by pigeons, Columba livia. Journal of the Experimental Analysis of Behavior, 57, 145-158.

Wilkie, D. M., Willson, R. J., \& Carr, J. A. R. (1999). Errors made by animals in memory paradigms are not always due to failures of memory. Neuroscience \& Biobehavioral Reviews, 23, 451-455.

(Manuscript received June 20, 2005;

revision accepted for publication December 13, 2005.) 